\title{
Increased Mortality Burden in Young Asian Subjects with Dysglycemia and Comorbidities
}

\author{
Eun-Jung Rhee ${ }^{1}$, Inha Jung ${ }^{1}$, Hyemi Kwon ${ }^{1}$, Se Eun Park ${ }^{1}$, Yang-Hyun Kim ${ }^{2}{ }^{\mathbb{D}}$, \\ Kyung-Do Han ${ }^{3}$, Yong-Gyu Park ${ }^{4, *,+}$ and Won-Young Lee ${ }^{1, *,+}$ (i)
}

1 Department of Endocrinology and Metabolism, Kangbuk Samsung Hospital, Sungkyunkwan University School of Medicine, 29 Saemunan-ro, Jongno-gu, Seoul 03181, Korea; hongsiri@hanmail.net (E.-J.R.); ina.jung@samsung.com (I.J.); hyemi.kwon@samsung.com (H.K.); seraph.park@samsung.com (S.E.P.)

2 Department of Family Medicine, Korea University Hospital, College of Medicine, Korea University, Seoul 02841, Korea; mrchir00@gmail.com

3 Department of Statistics and Actuarial Science, Soongsil University, 369 Sangdo-ro, Dongjak-gu, Seoul 06978, Korea; hkd917@naver.com

4 Department of Medical Statistics, Biomedicine \& Health Sciences, The Catholic University College of Medicine, 222 Banpo-daero, Seocho-gu, Seoul 06591, Korea

* $\quad$ Correspondence: ygpark@catholic.ac.kr (Y.G.P.); drlwy@hanmail.net (W.-Y.L.); Tel.: +82-2-596-4513 (Y.G.P.); +82-2-2001-2579 (W.-Y.L.)

$+\quad$ These authors contributed equally to the work reported.

Received: 3 March 2020; Accepted: 2 April 2020; Published: 7 April 2020

\begin{abstract}
Background: High blood glucose level has a linear relationship with all-cause mortality. However, the influence of glycemic abnormality on mortality differs by age group. We aimed to analyze all-cause mortality according to glycemic status, age groups, and comorbidities using a national health database. Methods: The 6,330,369 participants who underwent Korean National Health Screening in 2009 were followed up until 2016, with a median follow-up of 7.3 years. All-cause mortality rates were analyzed according to glycemic status (normoglycemia, impaired fasting glucose [IFG], newly diagnosed diabetes, diabetes duration $<5$ years, diabetes duration $\geq 5$ years), age groups (20-39, 40-65, and $\geq 65$ years), and comorbidities using the Korean National Health Insurance System database. Results: At baseline, 712,901 (11.3\%) subjects had diabetes. Compared with subjects without diabetes, those with diabetes at baseline showed increased mortality risk after adjustment for multiple risk factors (hazard ratio [HR] 1.613; 95\% confidence interval [CI] 1.598,1.629), and those with IFG showed a significantly increased mortality risk compared with normoglycemic subjects (HR 1.053; 95\% CI 1.042,1.064). Mortality risk associated with glycemic status decreased gradually from younger to older age groups and was consistently higher in those with diabetes with coronary heart disease, ischemic stroke or decreased renal function than those without comorbidities. Conclusion: Compared with normoglycemic subjects, subjects with diabetes and IFG had an increased mortality risk and the mortality risk was higher in the younger age group than in the older age group. The presence of diabetes and comorbid diseases synergistically increased mortality risk.
\end{abstract}

Keywords: prediabetes; diabetes mellitus; mortality; comorbidity; Korean National Health Insurance Corporation

\section{Introduction}

The prevalence of diabetes is increasing rapidly worldwide, especially in the Asia-Pacific region [1-3]. Although the life-expectancy of patients with diabetes has been prolonged, the increasing incidence of diabetes in young adults is a new serious trend worldwide and especially in the Asia-Pacific area [4]. The increased prevalence of young-onset diabetes has raised concerns because longer years of 
life were lost to diabetes, subsequently resulting in a poor quality of life. In addition, diabetes has been highly ranked as a cause of death globally over the last decade [5]. Since diabetes is a complex disease with multiple complications, the actual mortality attributable to diabetes could be higher.

Prediabetes is a condition that precedes diabetes and is defined by blood glucose levels higher than normoglycemia but not reaching the diabetic status [6]. Although diabetes and prediabetes are separate conditions that are defined by definite cutoff levels of blood glucose, these two conditions are considered as a continuum. Compared with normoglycemic subjects, subjects with the prediabetic status have an increased risk for cardiovascular and chronic kidney diseases [7-9]. In addition, mortality rate increases as the glycemic status deteriorates from normoglycemia to diabetes [10].

Diabetes causes multiple complications that increase the mortality rate [11,12]. In a recent study that included 271,174 patients with diabetes registered in the Swedish National Diabetes Register, who were matched with 1,355,870 controls, the relative importance of risk factors for predicting death and other cardiovascular diseases was significantly different, suggesting different effects of coexisting conditions on the outcome of these patients [13]. In addition, among various diabetic complications, the coexistence of which complications would shorten life expectancy more was not clearly defined.

Although the presence of diabetes affects life expectancy, the outcome differs according to age at the onset of diabetes. In a study comprising 318,083 patients with diabetes, who were matched with under 1.6 million controls and followed up for 5.6 years, patients who were diagnosed at younger ages showed significantly increased risks for most serious outcomes compared with the controls [14]. Interestingly, if the patient was diagnosed with diabetes at $>80$ years of age, the hazard ratio for the outcomes was $<1$, suggesting that by this age, survival in those with diabetes was the same as that in the controls. However, similar analyses were not performed in Asians.

In this study, using the Korean National Health Insurance System (NHIS) database, 6,330,369 participants who underwent Korean National Health Screening (KNHS) in 2009 were followed up until 2016, and all-cause mortality rates were analyzed according to baseline glycemic status. In addition, all-cause mortality risk was specifically analyzed according to baseline risk factors, comorbidities, and age groups.

\section{Methods}

\subsection{NHIS Database and NHIS Health Checkup Data}

Nearly all Koreans (97.2\% of the Korean population, approximately 50 million) are covered by the Korean NHIS, which is a nonprofit, single-payer organization by the Korean government. The NHIS maintains patients' demographic information, examinations data, claims for disease diagnosis codes of the International Classification of Diseases (ICD-10), and treatment records that can be used to produce a population-based cohort [15]. Insured Korean adults aged $>40$ years and employees aged $>20$ years undergo regular health checkups provided by the NHIS every 1 or 2 years.

There were two types of NHIS databases available to the researchers: one was a customized research database, composed of data from all Koreans, and the other was a sample research database, composed of data from a select group of one million Koreans. This study was performed using the customized research database that included all data available regarding our customized variables and follow-up years. Our analyses were performed after NHIS approval for the use of its database for this research (NHIS-2019-1-248).

Our study protocol was approved by the Institutional Review Board of the Kangbuk Samsung Hospital (KBSMC2019-01-034). The requirement for informed consent was waived as the data released to the researchers were de-identified.

\subsection{Measurements}

Body weight $(\mathrm{kg})$ and height $(\mathrm{cm})$ were measured using an electronic scale, and waist circumference $(\mathrm{WC} ; \mathrm{cm})$ was measured at the middle point between the rib cage and iliac crest by trained 
examiners. All blood samples were collected after fasting, and blood pressure was measured using a sphygmomanometer after $5 \mathrm{~min}$ of rest. Baseline health behaviors such as income, smoking, alcohol consumption, and exercise were confirmed using standardized questionnaires. Body mass index (BMI) was calculated as body weight $(\mathrm{kg})$ divided by height $(\mathrm{m})$ squared.

Participants were divided into three groups according to their smoking status (never smokers, ex-smokers, and current smokers) and into three groups according to their alcohol consumption status (non-drinkers, drinking $<30 \mathrm{~g} /$ day [moderate drinker], and drinking $\geq 30 \mathrm{~g} /$ day [heavy drinker]). Physical activity was defined as engaging in regular exercise (either of the following intensity levels): physical activity with high intensity for $>20 \mathrm{~min}$ per session $\geq 3$ days per week and physical activity with moderate intensity for $>30$ min per session $\geq 5$ days per week.

\subsection{Study Design and Definition of Diseases}

All participants included in KNHS from January 2009 to December $2009(n=10,505,818)$ were initially enrolled in the study. Participants younger than 20 years $(n=15,327)$ and those with missing data for baseline characteristics and covariates $(n=4,160,122)$ were subsequently excluded, and the number of final study population was 6,330,369.

The mortality status at the end of 2016 was assessed as the primary outcome. The risk of all-cause mortality was analyzed in groups categorized by baseline glycemic status assessed with respect to fasting blood glucose (FBG). Impaired fasting glucose (IFG) was defined as FBG of 100-125 mg/dL, and diabetes was defined as FBG $\geq 126 \mathrm{mg} / \mathrm{dL}$ or ICD-10 code E11-14 with a claim for anti-diabetic medication [6]. Newly developed diabetes was defined as FBG $\geq 126 \mathrm{mg} / \mathrm{dL}$ in KNHS in 2009 and no claim for ICD-10 code E11-14 or anti-diabetic medication before 2009. The duration of claims for the above codes with anti-diabetic medication prescription before 2009 was defined as the duration of diabetes.

In addition, the risk of all-cause mortality was analyzed according to baseline risk factors, disease status, and the presence or absence of diabetes. The BMI of the participants was divided into three levels $\left(<18.5,18.5-25\right.$ and $\left.>25 \mathrm{~kg} / \mathrm{m}^{2}\right)$, and the renal function of the participants was divided into four groups according to the baseline estimated glomerular filtration rate (eGFR), calculated using the Modification of Diet in Renal Disease method [16]. Ischemic heart disease was defined using the claims of ICD-10 codes I21-25, and ischemic stroke was defined using code I63 or I64. The recommended cutoff values in Koreans for abdominal obesity was defined as waist circumference $\geq 90 \mathrm{~cm}$ in men and $\geq 85 \mathrm{~cm}$ in women [17]. Dyslipidemia was defined by fasting total cholesterol $\geq 240 \mathrm{mg} / \mathrm{dL}$ or claims of ICD-10 codes E78 and lipid-lowering medications. Hypertension was defined by blood pressure $\geq$ 140/90 mmHg or ICD-10 codes I10-13, I15 and medications.

\subsection{Statistical Analysis}

Comparisons of the continuous variables at baseline between participants who were alive and dead in 2016 were performed using the Student's $t$-test. Comparisons of the categorical variables between the groups were performed using the chi-square test.

Hazard ratios (HRs) with 95\% confidence intervals (CIs) were assessed using the Cox proportional hazards model by analyzing mortality risk according to baseline glycemic status, and obesity status was assessed based on BMI or abdominal obesity. We conducted multivariable adjustments for age, sex, current smoking, alcohol drinking, regular exercise, body mass index, hypertension, dyslipidemia, and chronic kidney disease, which could affect outcomes. In addition, we performed subgroup analyses using the Cox proportional hazards model with $\mathrm{P}$ for interaction, according to the presence or absence of the various risk factors and diseases. Mortality rate ratio (MRR) was calculated with the incidence rate of patients with diabetes divided by that of patients without diabetes.

$P$ values $<0.05$ were considered statistically significant. SAS version 9.3 (SAS Institute Inc., Cary, NC, USA) was used for all statistical analyses. 


\section{Results}

Among 6,330,369 subjects who participated in KNHS in 2009, 712,901 (11.3\%) subjects had diabetes (Table 1). The mean age of the participants was 48.1 years, and $56.4 \%$ of subjects were men. The median follow-up period was 7.3 years. Those who had diabetes at baseline were older, more obese in general and abdominally, had higher baseline FBG levels, higher blood pressure, and had a higher proportion of cardiovascular diseases than those who did not have diabetes (Table 1).

Table 1. General characteristics of the participants according to baseline diabetes status.

\begin{tabular}{|c|c|c|c|}
\hline & $\begin{array}{l}\text { No Diabetes } \\
(n=5,617,468)\end{array}$ & $\begin{array}{c}\text { Diabetes } \\
(n=712,901)\end{array}$ & \\
\hline Age (years) & $46.9 \pm 14.4$ & $58.4 \pm 11.7$ & $<0.0001$ \\
\hline Sex: men $(\%)$ & $3,144,640(56.0)$ & $427,084(59.9)$ & $<0.0001$ \\
\hline Body weight (kg) & $64.1 \pm 11.6$ & $66.0 \pm 11.4$ & $<0.0001$ \\
\hline Body mass index $\left(\mathrm{kg} / \mathrm{m}^{2}\right)$ & $23.7 \pm 3.2$ & $25.1 \pm 3.3$ & $<0.0001$ \\
\hline Waist circumference $(\mathrm{cm})$ & $80.2 \pm 9.0$ & $85.6 \pm 8.4$ & $<0.0001$ \\
\hline Fasting blood glucose (mg/dL) & $92.9 \pm 11.4$ & $144.3 \pm 45.8$ & $<0.0001$ \\
\hline Total cholesterol (mg/dL) & $194.9 \pm 36.1$ & $195.2 \pm 41.3$ & $<0.0001$ \\
\hline Systolic blood pressure (mmHg) & $122.5 \pm 14.9$ & $129.2 \pm 15.7$ & $<0.0001$ \\
\hline Diastolic blood pressure (mmHg) & $76.5 \pm 9.9$ & $78.9 \pm 10.1$ & $<0.0001$ \\
\hline Obesity $\left(\mathrm{BMI} \geq 25 \mathrm{~kg} / \mathrm{m}^{2}\right)(\%)$ & $1,809,615(32.2)$ & $340,975(47.8)$ & $<0.0001$ \\
\hline Dyslipidemia (\%) & $1,000,705(17.8)$ & $305,720(42.9)$ & $<0.0001$ \\
\hline Hypertension (\%) & $1,532,538(27.28)$ & $434,475(60.94)$ & $<0.0001$ \\
\hline Chronic kidney disease (\%) & $354,747(6.3)$ & $91,858(12.9)$ & $<0.0001$ \\
\hline Heart disease (\%) & $156,214(2.8)$ & $41,191(5.8)$ & $<0.0001$ \\
\hline Ischemic stroke (\%) & $83,069(1.5)$ & $18,541(2.6)$ & $<0.0001$ \\
\hline Current smoker (\%) & $1,480,548(26.4)$ & $174,548(24.5)$ & $<0.0001$ \\
\hline Heavy alcohol drinker (\%) & $380,422(6.8)$ & $57,953(8.1)$ & $<0.0001$ \\
\hline Regular exercise (\%) & $2,902,134(51.7)$ & $348,223(48.9)$ & $<0.0001$ \\
\hline
\end{tabular}

Values are expression as mean \pm standard deviations or number $(\%)$.

When the HR of mortality was analyzed according to baseline glycemic status, compared with subjects without diabetes, those with diabetes at baseline showed 1.6-fold increased mortality risk after adjustment for multiple risk factors (Table 2). When the mortality risk was analyzed according to baseline glycemic status, compared with normoglycemic subjects, subjects with IFG showed weak but significantly increased risk for mortality. Compared with normoglycemic subjects, subjects with newly developed diabetes showed 1.44-fold increased mortality risk, and the mortality risk gradually increased up to 1.77-fold as the duration of diabetes lengthened to $\geq 5$ years (Table 2). Similar findings were observed when the analyses were performed separately in a different sex.

To evaluate the effect of various diseases in association with diabetes on mortality risk, separate analyses were performed based on the presence and absence of various diseases and risk factors in association with diabetes (Table 3, Supplemental Table S1). The mortality risk was consistently higher in subjects with diabetes than in those without diabetes, although the subjects had ischemic heart disease or ischemic stroke (Table 3, Figure 1). The mortality risk gradually increased when eGFR of the subjects decreased from normal to $<45 \mathrm{~mL} / \mathrm{min} / 1.73 \mathrm{~m}^{2}$. Subjects with diabetes and eGFR $<45$ $\mathrm{mL} / \mathrm{min} / 1.73 \mathrm{~m}^{2}$ had the highest mortality risk among the subjects divided on the basis of eGFR (3.385, 95\% confidence interval [CI] 3.292-3.481), and this risk was the highest among the risks calculated for various diseases and risk factors associated with diabetes, suggesting that decreased renal function was the strongest risk factor to cause mortality in subjects with diabetes (Table 3, Figure 1). 
Table 2. IR and multivariate-adjusted HR of mortality according to baseline glycemic status and diabetes duration.

\begin{tabular}{|c|c|c|c|c|c|}
\hline \multirow{2}{*}{ Glycemic Status } & \multirow{2}{*}{ Total Number } & \multirow{2}{*}{ Number of Events } & \multirow{2}{*}{$\begin{array}{c}\text { IR } \\
\text { (Per } 1000 \text { Person Years) }\end{array}$} & \multicolumn{2}{|c|}{ Multivariate-Adjusted HR (95\% CI) } \\
\hline & & & & Model 1 & Model 2 \\
\hline \multicolumn{6}{|l|}{ Diabetes } \\
\hline No & $5,617,468$ & 157,328 & 0.384 & 1.000(reference) & 1000(reference) \\
\hline Yes & 712,901 & 63,298 & 1.244 & $1.547(1.533,1.561)$ & $1.613(1.598,1.629)$ \\
\hline \multicolumn{6}{|l|}{ Glycemic status } \\
\hline No diabetes & $4,176,024$ & 103,156 & 0.338 & 1.000(reference) & 1000(reference) \\
\hline Impaired fasting glucose & $1,427,415$ & 52,650 & 0.507 & $1.013(1.002,1.024)$ & $1.053(1.042,1.064)$ \\
\hline Newly developed diabetes & 186,940 & 10,903 & 0.812 & $1.398(1.371,1.426)$ & $1.438(1.41,1.467)$ \\
\hline Diabetes duration $<5$ years & 260,228 & 21,608 & 1.157 & $1.467(1.446,1.489)$ & $1.594(1.57,1.618)$ \\
\hline Diabetes duration $\geq 5$ years & 279,762 & 32,309 & 1.635 & $1.691(1.670,1.712)$ & $1.774(1.752,1.797)$ \\
\hline \multicolumn{6}{|c|}{ Men } \\
\hline \multicolumn{6}{|l|}{ Diabetes } \\
\hline No & $3,144,640$ & 102,064 & 4.459 & 1.000(reference) & 1000(reference) \\
\hline Yes & 427,084 & 41,608 & 13.764 & $1.529(1.513,1.548)$ & $1.605(1.585,1.623)$ \\
\hline \multicolumn{6}{|l|}{ Glycemic status } \\
\hline No diabetes & $2,238,583$ & 65,487 & 4.012 & 1.000(reference) & 1000(reference) \\
\hline Impaired fasting glucose & 898,198 & 35,626 & 5.474 & $0.996(0.983,1.009)$ & $1.044(1.031,1.058)$ \\
\hline Newly developed diabetes & 132,313 & 7853 & 8.286 & $1.37(1.339,1.403)$ & $1.424(1.39,1.457)$ \\
\hline Diabetes duration $<5$ years & 149,612 & 14,442 & 13.589 & $1.492(1.465,1.519)$ & $1.633(1.603,1.663)$ \\
\hline Diabetes duration $\geq 5$ years & 153,018 & 20,264 & 18.982 & $1.63(1.604,1.656)$ & $1.731(1.703,1.759)$ \\
\hline \multicolumn{6}{|c|}{ Women } \\
\hline \multicolumn{6}{|l|}{ Diabetes } \\
\hline No & $2,472,828$ & 55,264 & 3.049 & 1.000(reference) & 1000(reference) \\
\hline Yes & 285,817 & 21,690 & 10.503 & $1.567(1.543,1.592)$ & $1.629(1.603,1.656)$ \\
\hline \multicolumn{6}{|l|}{ Glycemic status } \\
\hline No diabetes & $1,937,441$ & 37,669 & 2.651 & 1.000(reference) & 1000(reference) \\
\hline Impaired fasting glucose & 529,217 & 17,024 & 4.398 & $1.031(1.013,1.05)$ & $1.061(1.042,1.081)$ \\
\hline Newly developed diabetes & 54,627 & 3050 & 7.723 & $1.403(1.352,1.456)$ & $1.445(1.392,1.499)$ \\
\hline Diabetes duration $<5$ years & 110,616 & 7166 & 8.897 & $1.404(1.369,1.44)$ & $1.514(1.476,1.554)$ \\
\hline Diabetes duration $\geq 5$ years & 126,744 & 12,045 & 13.252 & $1.791(1.755,1.829)$ & $1.853(1.814,1.892)$ \\
\hline
\end{tabular}

Model 1: Adjusted for age and sex. Model 2: Adjusted for age, sex, current smoking, alcohol drinking, regular exercise, body mass index, hypertension, dyslipidemia and chronic kidney disease. IR, incidence rate; $\mathrm{HR}$, hazard ratio; $\mathrm{CI}$, confidence interval. 
Table 3. The impact of various diseases and risk factors on the association of mortality risk and diabetes.

\begin{tabular}{|c|c|c|c|c|c|c|}
\hline \multirow{2}{*}{ Diabetes Status } & \multirow{2}{*}{ Comorbidities } & \multirow{2}{*}{$\begin{array}{l}\text { Total } \\
\text { Number }\end{array}$} & \multirow{2}{*}{$\begin{array}{l}\text { Number } \\
\text { of Events }\end{array}$} & \multirow{2}{*}{$\begin{array}{c}\text { IR } \\
\text { (Per } 1000 \text { Person Years) }\end{array}$} & \multicolumn{2}{|c|}{ Multivariate-Adjusted HR (95\% CI) } \\
\hline & & & & & Model 1 & Model 2 \\
\hline \multicolumn{7}{|c|}{ Coronary heart disease } \\
\hline \multirow[t]{2}{*}{ No } & No & $5,461,254$ & 144,092 & 0.361 & 1.000(reference) & 1.000 (reference) \\
\hline & Yes & 156,214 & 13,236 & 1.180 & $1.293(1.27,1.317)$ & $1.35(1.326,1.374)$ \\
\hline \multirow[t]{3}{*}{ Yes } & No & 671,710 & 57,031 & 1.188 & $1.552(1.537,1.568)$ & $1.417(1.397,1.437)$ \\
\hline & Yes & 41,191 & 6267 & 2.185 & $1.875(1.828,1.924)$ & $1.737(1.69,1.787)$ \\
\hline & Ischemic stroke & & & & & \\
\hline \multirow[t]{2}{*}{ No } & No & $5,534,399$ & 149,816 & 0.371 & 1.000(reference) & 1.000 (reference) \\
\hline & Yes & 83,069 & 7512 & 1.266 & $1.582(1.546,1.619)$ & $1.592(1.556,1.63)$ \\
\hline \multirow[t]{2}{*}{ Yes } & No & 694,360 & 59,704 & 1.203 & $1.545(1.53,1.56)$ & $1.406(1.387,1.426)$ \\
\hline & Yes & 18,541 & 3594 & 2.847 & $2.402(2.324,2.483)$ & $2.144(2.07,2.221)$ \\
\hline \multicolumn{7}{|c|}{ Estimated glomerular filtration rate $\left(\mathrm{mL} / \mathrm{min} / 1.73 \mathrm{~m}^{2}\right)$} \\
\hline \multirow[t]{4}{*}{ No } & $>90$ & $2,117,700$ & 41,705 & 0.269 & 1.000(reference) & 1.000(reference) \\
\hline & $60-90$ & $3,145,021$ & 89,280 & 0.389 & $0.877(0.867,0.887)$ & $0.928(0.917,0.939)$ \\
\hline & $45-59$ & 215,123 & 19,038 & 1.233 & $1.112(1.093,1.132)$ & $1.204(1.183,1.226)$ \\
\hline & $<45$ & 139,624 & 7305 & 0.720 & $1.591(1.552,1.632)$ & $1.682(1.641,1.725)$ \\
\hline \multirow[t]{4}{*}{ Yes } & $>90$ & 229,022 & 14,473 & 0.878 & $1.52(1.492,1.549)$ & $1.432(1.402,1.463)$ \\
\hline & $60-90$ & 392,021 & 32,351 & 1.152 & $1.296(1.277,1.315)$ & $1.274(1.252,1.297)$ \\
\hline & $45-59$ & 66,923 & 10,624 & 2.278 & $1.649(1.614,1.685)$ & $1.622(1.583,1.663)$ \\
\hline & $<45$ & 24,935 & 5850 & 3.523 & $3.001(2.919,3.085)$ & $2.886(2.799,2.975)$ \\
\hline
\end{tabular}


Table 3. Cont.

\begin{tabular}{|c|c|c|c|c|c|c|}
\hline \multirow{2}{*}{ Diabetes Status } & \multirow{2}{*}{ Comorbidities } & \multirow{2}{*}{$\begin{array}{c}\text { Total } \\
\text { Number }\end{array}$} & \multirow{2}{*}{$\begin{array}{l}\text { Number } \\
\text { of Events }\end{array}$} & \multirow{2}{*}{$\begin{array}{c}\text { IR } \\
\text { (Per } 1000 \text { Person Years) }\end{array}$} & \multicolumn{2}{|c|}{ Multivariate-Adjusted HR (95\% CI) } \\
\hline & & & & & Model 1 & Model 2 \\
\hline \multicolumn{7}{|c|}{ Smoking } \\
\hline \multirow[t]{2}{*}{ No } & No & $4,136,920$ & 115,403 & 0.382 & 1.000 (reference) & 1.000(reference) \\
\hline & Current & $1,480,548$ & 41,925 & 0.389 & $1.672(1.652,1.692)$ & $1.545(1.526,1.564)$ \\
\hline \multirow[t]{2}{*}{ Yes } & No & 538,353 & 47,401 & 1.231 & $1.56(1.543,1.577)$ & $1.402(1.381,1.423)$ \\
\hline & Current & 174,548 & 15,897 & 1.286 & $2.522(2.479,2.565)$ & $2.157(2.115,2.2)$ \\
\hline \multicolumn{7}{|c|}{ Regular exercise } \\
\hline \multirow[t]{2}{*}{ No } & No & $2,715,334$ & 99,493 & 0.504 & 1.000 (reference) & 1.000(reference) \\
\hline & Yes & $2,902,134$ & 57,835 & 0.272 & $0.756(0.749,0.764)$ & $0.786(0.777,0.794)$ \\
\hline \multirow[t]{2}{*}{ Yes } & No & 364,678 & 40,383 & 1.565 & $1.571(1.553,1.59)$ & $1.42(1.398,1.442)$ \\
\hline & Yes & 348,223 & 22,915 & 0.914 & $1.154(1.137,1.171)$ & $1.073(1.054,1.093)$ \\
\hline \multicolumn{7}{|c|}{ Abdominal obesity (Men: $90 \mathrm{~cm} /$ Women: $85 \mathrm{~cm}$ ) } \\
\hline \multirow[t]{2}{*}{ No } & No & $4,121,743$ & 105,470 & 0.351 & 1.000(reference) & 1.000(reference) \\
\hline & Yes & $1,495,725$ & 51,858 & 0.475 & $0.862(0.853,0.871)$ & 1.197(1.182,1.213) \\
\hline \multirow[t]{2}{*}{ Yes } & No & 364,402 & 34,230 & 1.323 & $1.591(1.571,1.61)$ & $1.42(1.397,1.443)$ \\
\hline & Yes & 348,499 & 29,068 & 1.162 & $1.349(1.331,1.366)$ & $1.619(1.589,1.649)$ \\
\hline \multicolumn{7}{|c|}{ Body mass index $\left(\mathrm{kg} / \mathrm{m}^{2}\right)$} \\
\hline \multirow[t]{3}{*}{ No } & $<18.5$ & $2,406,712$ & 77,986 & 0.445 & $1.446(1.428,1.464)$ & $1.412(1.395-1.430)$ \\
\hline & $18.5-25$ & $1,401,141$ & 36,030 & 0.352 & 1.000(reference) & 1.000(reference) \\
\hline & $25-$ & $1,809,615$ & 43,312 & 0.327 & $0.965(0.952,0.979)$ & $0.957(0.943-0.970)$ \\
\hline \multirow[t]{3}{*}{ Yes } & $<18.5$ & 188,227 & 25,411 & 1.934 & $2.443(2.404,2.483)$ & $2.030(1.991-2.069)$ \\
\hline & $18.5-24.9$ & 183,699 & 15,523 & 1.180 & $1.63(1.6,1.661)$ & $1.375(1.345-1.405)$ \\
\hline & $\geq 25$ & 340,975 & 22,364 & 0.909 & $1.495(1.47,1.52)$ & $1.262(1.237-1.286)$ \\
\hline
\end{tabular}

Model 1: Adjusted for age and sex. Model 2: Adjusted for age, sex, current smoking, alcohol drinking, regular exercise, body mass index, hypertension, dyslipidemia, chronic kidney disease and diabetes duration. IR, incidence rate, HR, hazard ratio; CI, confidence interval. 


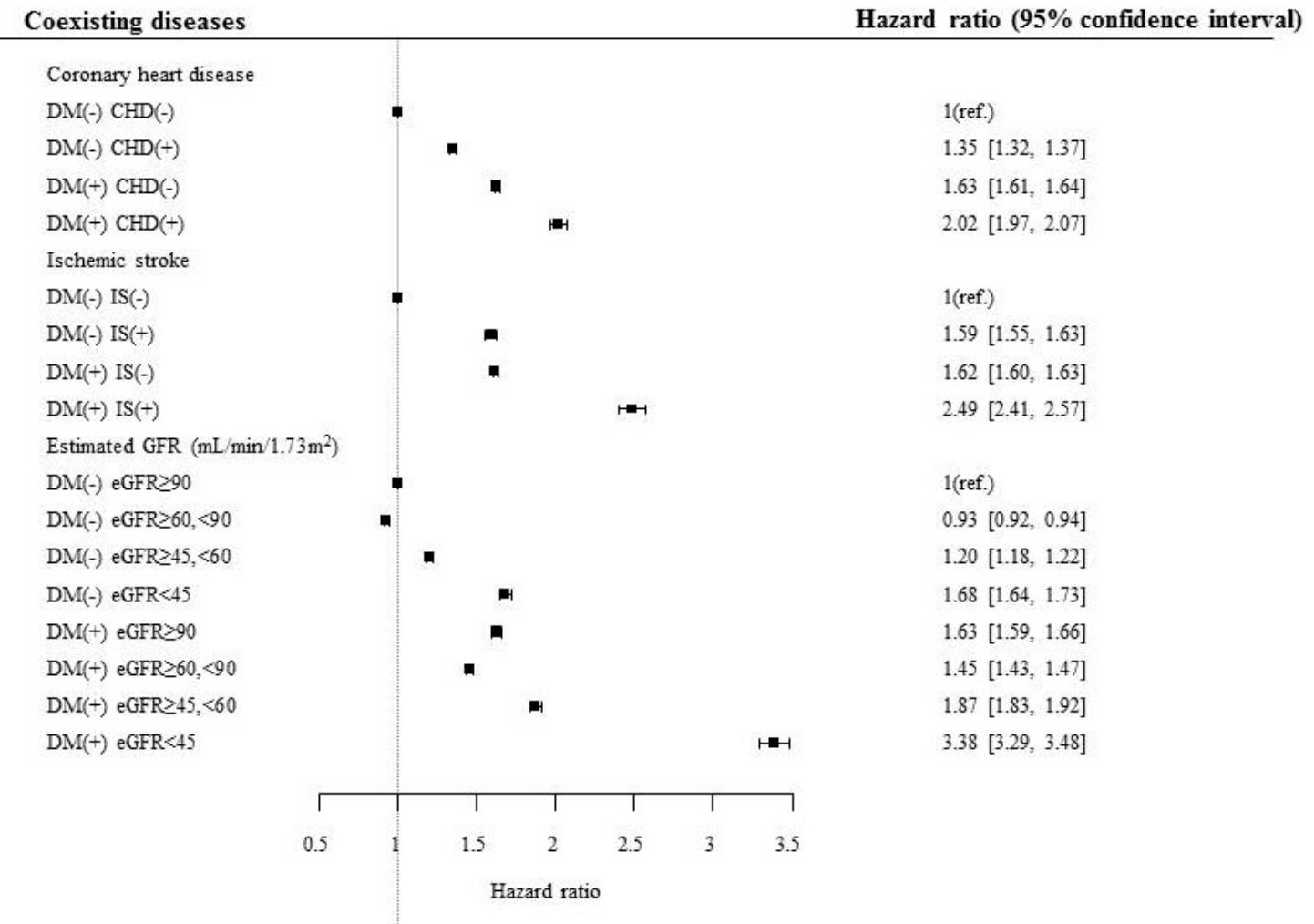

Figure 1. Hazard ratio for mortality according to the presence of diabetes, coronary heart disease, and ischemic stroke. DM, diabetes mellitus; CHD, coronary heart disease; IS, ischemic stroke; eGFR, estimated glomerular filtration rate.

The subjects had an increased mortality risk when they currently smoked, did not exercise, or had abdominal obesity, and these risks were higher when the subjects had diabetes than when they did not. Interestingly, compared with subjects with normal weight, those who were underweight showed significantly increased mortality risk, and this finding was also prominent in those with diabetes, with underweight diabetes subjects showing 2.3-fold increased mortality risk compared with non-diabetes, normal weight subjects (Table 3 ). Similar results were observed when these analyses were performed in different sexes (Supplementary Table S1).

When the above analyses were performed in different age groups, somewhat different trends were observed (Supplementary Table S2). In the age group of 20-39 years, the highest mortality risk was observed in those who had ischemic stroke and diabetes among the various diseases and risk factors (4.266, 95\% CI 1.604-11.349). However, in the age group of $\geq 65$ years, those who had eGFR $<45 \mathrm{~mL} / \mathrm{min} / 1.73 \mathrm{~m}^{2}$ showed the highest mortality risk among the various diseases and risk factors (3.245, 95\% CI 3.14-3.353), suggesting varying influence of comorbid conditions on mortality risk in subjects with diabetes in different age groups (Supplementary Table S2).

When the incidence rate of mortality and MRR was analyzed according to different age groups, the MRR associated with diabetes decreased gradually from younger to older age groups (Table 4, Figure 2). For example, the patients with diabetes who were aged $\geq 90$ years at baseline had approximately 1.2 -fold increased mortality risk compared with those without diabetes in the same age group. In contrast, patients with diabetes who were aged 40 years showed 3.04-fold increased mortality compared with those without diabetes in the same age group (Figure 2A, Supplementary Table S3). 
Table 4. IR and multivariate-adjusted HR of mortality according to baseline glycemic status in different age groups.

\begin{tabular}{|c|c|c|c|c|c|}
\hline \multirow{2}{*}{ Glycemic Status } & \multirow{2}{*}{ Total Number } & \multirow{2}{*}{ Number of Events } & \multirow{2}{*}{$\begin{array}{c}\text { IR } \\
\text { (Per 1000 Person Years) }\end{array}$} & \multicolumn{2}{|c|}{ Multivariate-Adjusted HR (95\% CI) } \\
\hline & & & & Model 1 & Model 2 \\
\hline \multicolumn{6}{|c|}{ 20-39 years } \\
\hline \multicolumn{6}{|l|}{ Diabetes } \\
\hline No & $1,877,765$ & 6589 & 0.478 & 1.000(reference) & 1.000 (reference) \\
\hline Yes & 42,780 & 375 & 1.204 & $2.066(1.859,2.294)$ & $1.845(1.658,2.053)$ \\
\hline \multicolumn{6}{|l|}{ Glycemic status } \\
\hline No diabetes & $1,563,198$ & 5296 & 0.461 & 1.000(reference) & 1.000(reference) \\
\hline Impaired fasting glucose & 314,256 & 1291 & 0.562 & $1.063(1,1.13)$ & $1.03(0.968,1.096)$ \\
\hline Newly developed diabetes & 29,237 & 232 & 1.089 & $1.923(1.685,2.194)$ & $1.735(1.518,1.983)$ \\
\hline Diabetes duration $<5$ years & 10,356 & 106 & 1.405 & $2.375(1.958,2.881)$ & $2.062(1.693,2.511)$ \\
\hline Diabetes duration $\geq 5$ years & 3498 & 39 & 1.538 & $2.596(1.894,3.56)$ & $2.249(1.637,3.088)$ \\
\hline \multicolumn{6}{|c|}{$40-64$ years } \\
\hline \multicolumn{6}{|l|}{ Diabetes } \\
\hline No & $2,988,010$ & 49,782 & 2.273 & 1.000(reference) & 1.000 (reference) \\
\hline Yes & 434,801 & 18,641 & 5.915 & $1.838(1.808,1.869)$ & $1.845(1.812,1.88)$ \\
\hline \multicolumn{6}{|l|}{ Glycemic status } \\
\hline No diabetes & $2,117,487$ & 32,457 & 2.089 & 1.000(reference) & 1.000(reference) \\
\hline Impaired fasting glucose & 862,911 & 17,010 & 2.696 & $1.063(1.044,1.084)$ & $1.09(1.069,1.11)$ \\
\hline Newly developed diabetes & 121,376 & 3963 & 4.507 & $1.589(1.537,1.643)$ & $1.589(1.537,1.643)$ \\
\hline Diabetes duration $<5$ years & 164,923 & 6875 & 5.730 & $1.853(1.805,1.902)$ & $1.931(1.879,1.983)$ \\
\hline Diabetes duration $\geq 5$ years & 156,114 & 8118 & 7.197 & $2.1(2.049,2.153)$ & $2.104(2.051,2.158)$ \\
\hline \multicolumn{6}{|c|}{$\geq 65$ years } \\
\hline \multicolumn{6}{|l|}{ Diabetes } \\
\hline No & 751,693 & 100,957 & 18.929 & 1.000(reference) & 1.000 (reference) \\
\hline Yes & 235,320 & 44,282 & 27.251 & $1.488(1.471,1.504)$ & $1.553(1.536,1.57)$ \\
\hline \multicolumn{6}{|l|}{ Glycemic status } \\
\hline No diabetes & 495,339 & 65,403 & 18.583 & 1.000(reference) & 1.000(reference) \\
\hline Impaired fasting glucose & 250,248 & 34,349 & 19.381 & $1.004(0.991,1.017)$ & $1.043(1.029,1.057)$ \\
\hline Newly developed diabetes & 36,327 & 6708 & 26.800 & $1.314(1.281,1.347)$ & $1.357(1.323,1.391)$ \\
\hline Diabetes duration $<5$ years & 84,949 & 14,627 & 24.668 & $1.382(1.357,1.407)$ & $1.499(1.472,1.527)$ \\
\hline Diabetes duration $\geq 5$ years & 120,150 & 24,152 & 29.333 & $1.635(1.611,1.659)$ & $1.716(1.69,1.742)$ \\
\hline
\end{tabular}

Model 1: Adjusted for age and sex. Model 2: Adjusted for age, sex, current smoking, alcohol drinking, regular exercise, body mass index, hypertension, dyslipidemia and chronic kidney disease. IR, incidence rate; HR, hazard ratio. 
25

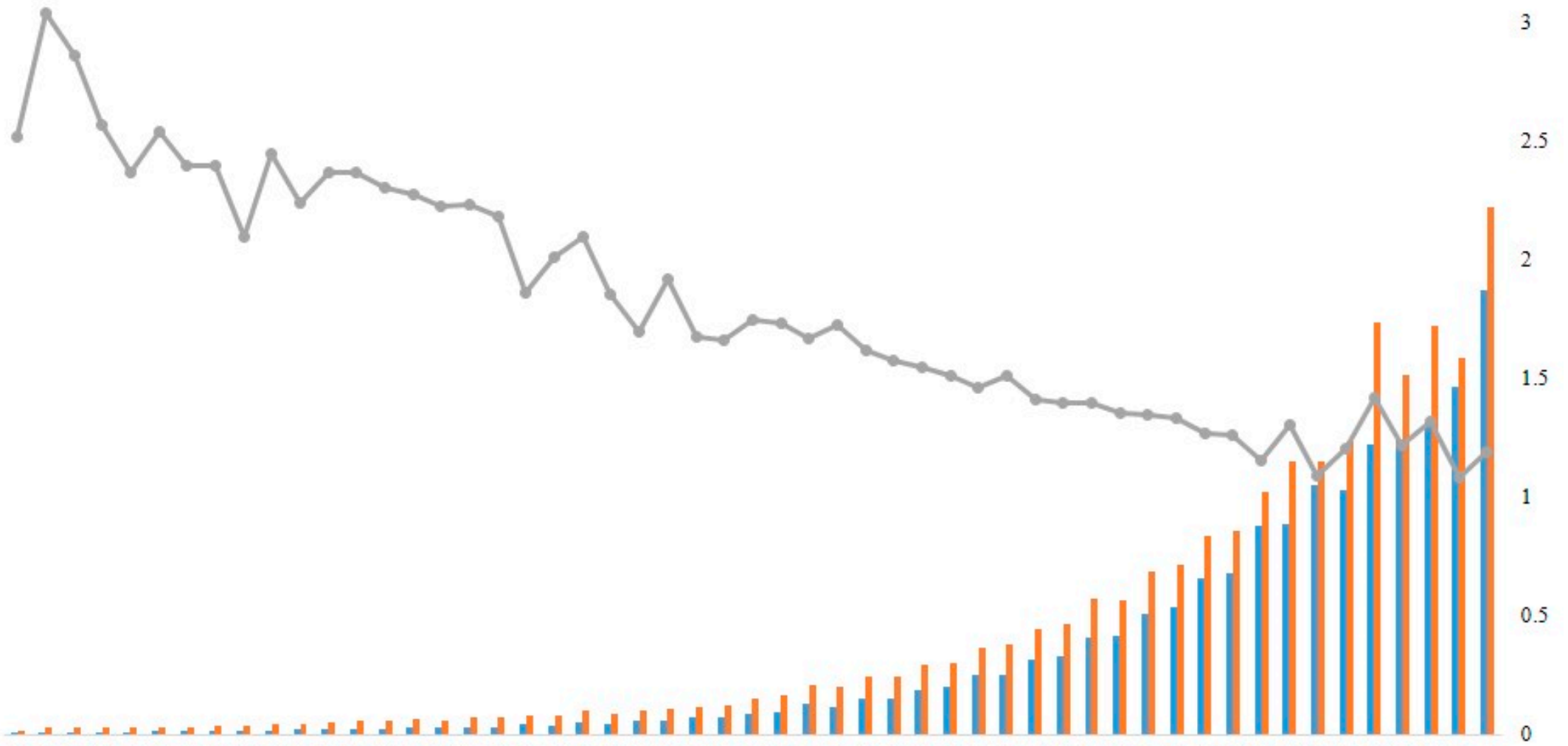

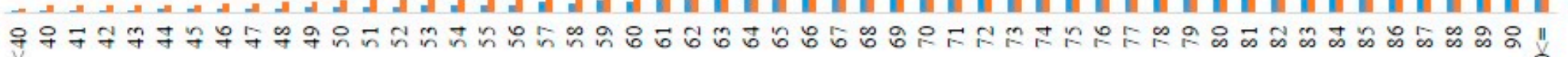

Figure 2. Cont. 

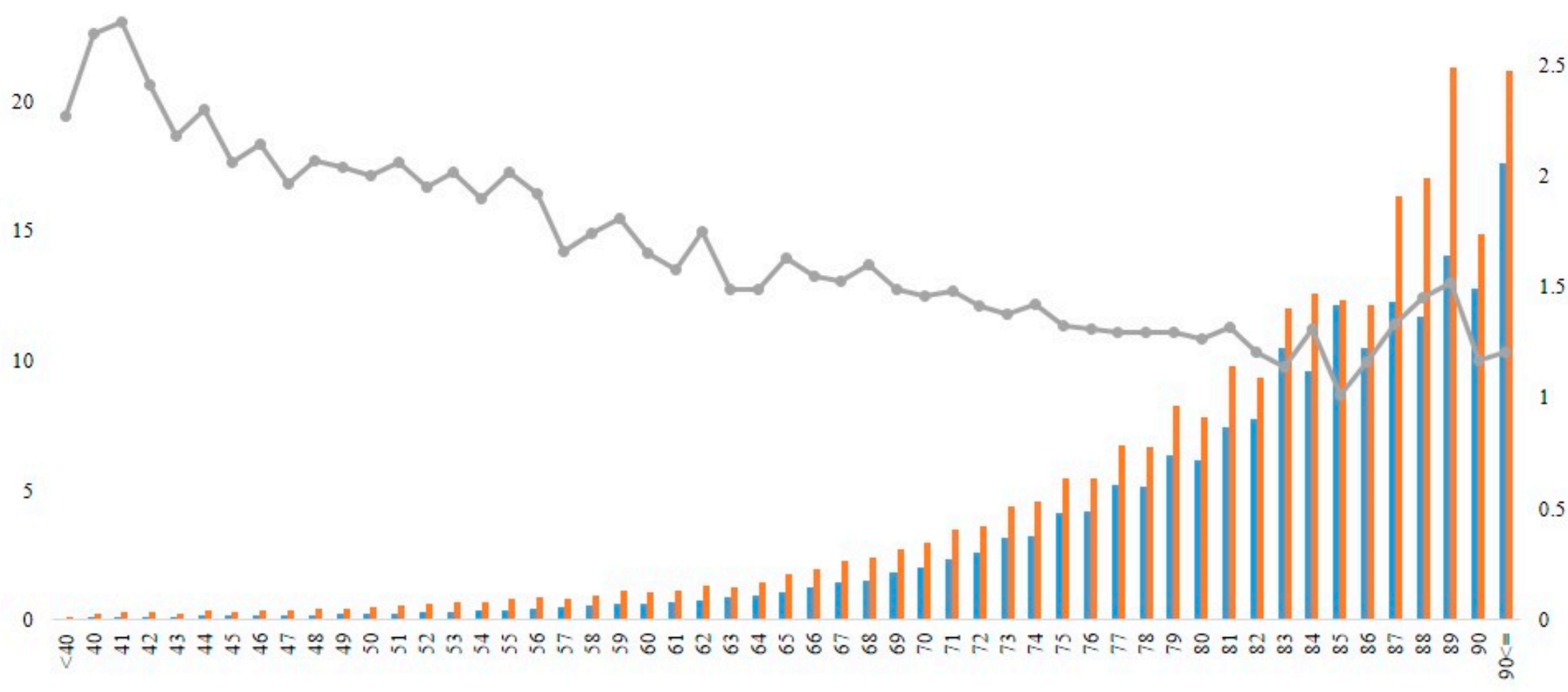$$
\text { - NoDM }=\mathrm{DM} \simeq \mathrm{MRR}
$$$$
\text { (B) }
$$

Figure 2. Cont. 
20

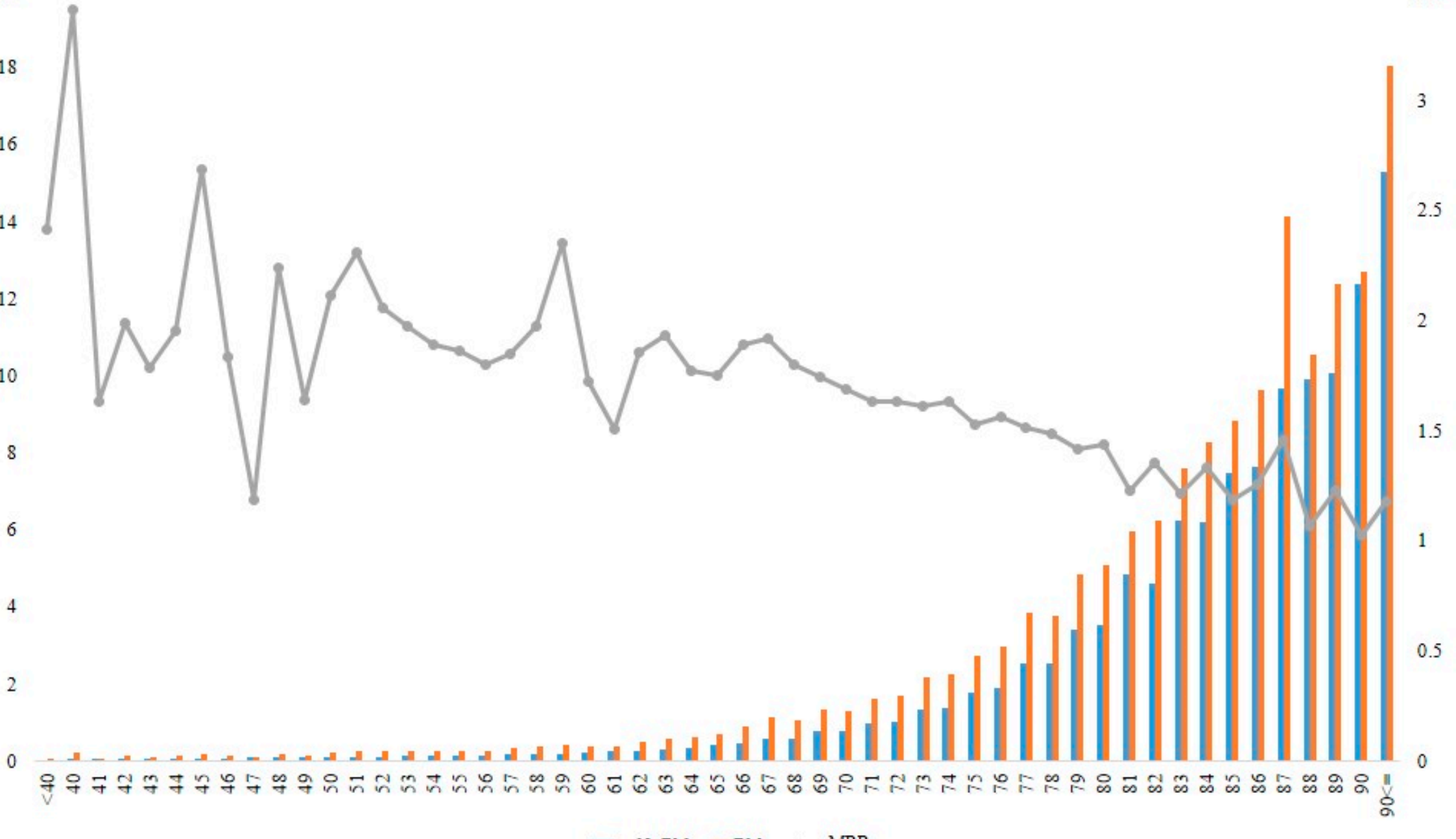$$
\text { - NoDM }=\mathrm{DM}=\mathrm{MRR}
$$

(C)

Figure 2. Incidence rate and MRR according to age groups in total (A), in men (B), and in women (C). DM, diabetes mellitus; MRR, mortality rate ratio. 


\section{Discussion}

In this large nationwide health screening and insurance database, compared with normoglycemic subjects and those with prediabetes, subjects with abnormal glucose levels had significantly increased mortality risks. Compared with non-diabetes subjects, subjects with diabetes at baseline showed 1.6-fold increased mortality risk, and the risk gradually increased as the duration of diabetes lengthened. In addition, although the presence of various diseases and metabolic risk factors increased mortality significantly, the presence of diabetes potentiated the risk across the diseases and risk factors. Among various comorbid conditions, renal dysfunction with an eGFR $<45 \mathrm{~mL} / \mathrm{min} / 1.73 \mathrm{~m}^{2}$ showed the highest mortality risk in subjects with diabetes, and this trend was somewhat different in different age groups. When the analyses for mortality risk were performed in different age groups, mortality risk gradually decreased from younger to older age groups, suggesting a higher burden of diabetes-related mortality risk in younger subjects than in older subjects.

Increased mortality risk in patients with diabetes has long been a great burden on the health professionals caring for them. Diabetes has been ranked as the $7^{\text {th }}$ leading cause of death, in the annual report of World Health Organization, although the actual rank could be higher as diabetes is a risk factor for other causes of death, such as cardiovascular disease [5]. In Korea, diabetes is the leading cause of death, and this has been observed in other Asia-Pacific regions as well [18,19]. In our study, subjects with diabetes showed 1.6-fold increased mortality risk compared with non-diabetic Korean adults after analyzing the nationwide health screening and claim data of 6,330,369 participants; the mortality risk gradually increased as the duration of diabetes increased from newly developed to $<5$ years to $\geq 5$ years.

In our study, compared with normoglycemic subjects, subjects with IFG, defined by fasting glucose between 100 and $125 \mathrm{mg} / \mathrm{dL}$ in health examinations, showed weak but significantly increased mortality risk with a HR of 1.053. Although this was a small increase, it was meaningful as the mortality risk was assessed in a large nationwide cohort. In a recent meta-analysis with 1.6 million participants, prediabetes increased the mortality risk by 1.13-fold, similar to our analysis [7]. Although the absolute excess risk was small, the fact that mortality risk actually increased in the subjects with prediabetes warrants early interventions and lifestyle modifications in these subjects.

In the Steno- 2 trial, multifactorial intervention in type 2 diabetes patients showed a $53 \%$ reduction in cardiovascular events compared with conventional intervention in type 2 diabetes patients, strongly suggesting the importance of controlling multiple risk factors for the prevention of cardiovascular diseases [20]. In the analyses from Emerging Risk Factors Collaboration, the HRs for all-cause mortality in those with diabetes were 1.9 compared with those without diabetes [21]. However, this risk increased to 3.7 or 3.8 if the subjects had diabetes and myocardial infarction or stroke simultaneously, suggesting increased mortality in patients with diabetes along with cardiometabolic multimorbidity. In addition, recent results from large cardiovascular outcome trials of novel anti-diabetic agents that control not only glucose but also multiple metabolic risk factors, reconfirms the importance of simultaneous intervention of multiple risk factors in patients with diabetes for the prevention of cardiovascular diseases [22]. Our study results showed that decreased renal function with eGFR $<45 \mathrm{~mL} / \mathrm{min} / 1.73 \mathrm{~m}^{2}$ resulted in a 3.4-fold increased mortality risk in patients with diabetes, which is the highest among the various comorbidities. However, younger groups with ischemic stroke and diabetes showed the highest mortality risk among the comorbidities, significantly different from the older age groups, which showed the highest mortality risk in the low eGFR group, similar to the findings of the overall study. These results suggest different influences of individual comorbidities on mortality, in various age groups.

Recent data report an increased incidence of young-onset diabetes [4]. In our study, increased mortality observed in patients with diabetes decreased gradually from younger to older age groups. For example, patients with diabetes who were aged $\geq 90$ years at baseline had approximately 1.2 -fold increased mortality risk compared with those without diabetes in the same age group. In contrast, patients with diabetes who are aged 40 years showed 3.04-fold increased mortality compared with 
those without diabetes in the same age group. These results suggest that there may be greater potential gains from more aggressive treatments in younger patients than in older patients with diabetes. Similar results were observed in previous studies from other ethnic groups. In a cohort study using Clinical Practice Research Datalink data from 383 general practices in England, it was found that in 187,968 patients with incident type 2 diabetes, the life expectancy differences between subjects with and without diabetes declined with age attained [23]. In the Swedish National Diabetes Registry, those who were diagnosed with diabetes at $\leq 40$ years had 2-fold excess mortality risk relative to the controls, and this risk attenuated progressively with each increasing decade of age, similar to our study results [14]. Our results as well as previous studies suggest that the mortality risk associated with diabetes differs markedly with age at diagnosis and presumably by ethnicity, with the highest mortality risk observed in those with early diagnosed diabetes.

In our study, compared with subjects with normal weight, those who were underweight showed significantly increased mortality risk, and this finding was also prominent in those with diabetes. Furthermore, non-diabetic obese subjects showed decreased mortality risk compared with subjects with normal body weight. In a recent study from the Taiwan Diabetes Study, a population-based retrospective cohort study, the highest hospitalization and mortality rate was observed among patients with BMI $<18.5 \mathrm{~kg} / \mathrm{m}^{2}$ among groups categorized according to BMI [24]. In another study performed in sample cohorts of Korean NHIS, diabetes-related mortality risk was higher in adults with lower BMI relative to those with higher BMI, similar to the results of our study [25]. Several possible explanations exist for these results: (1) sarcopenia reflected by lower BMI in patients with diabetes is related to increased mortality [26], (2) sarcopenic obesity with reduced muscle mass and high body fat frequently associated with patients with type 2 diabetes is known be associated with increased mortality [27], (3) being underweight might mean having a lower metabolic reserve that could protect against poor prognosis or outcomes, and (4) patients with type 2 diabetes incident at a low BMI due to genetic susceptibility have worse prognosis compared with those who developed type 2 diabetes due to the metabolic stress of obesity, in whom diabetes could disappear if they lost weight [28,29]. However, the precise mechanism for increased mortality in underweight subjects is not clearly defined.

Our study has limitations. First, the definition of glycemic abnormality was evaluated only with FBG levels; HbA1c values were not available in the analyses. Therefore, there could be some inaccuracies regarding allocation of the participants. Second, definitions of the diseases were evaluated only with claim records and not with actual diagnosis. However, claim data are presumably accurate in most of the diseases. Third, since the cause of death could not be verified due to the inability to link data for cause of death with NHIS data, only the all-cause mortality rate was analyzed. Fourth, diagnosis and definition of prediabetes and diabetes could not be made due to the lack of $\mathrm{HbA1c}$ data in national examinations. Fifth, at least in this study, we could not include the specific data for anti-diabetic medications in the analyses. There are possibilities for biases caused by different medication usage. Despite these limitations, this study is meaningful in that, the association of diabetes, age, and comorbid diseases with mortality, were analyzed in a large nationwide database.

In conclusion, our study showed that compared with normoglycemic subjects, subjects with prediabetes had an increased mortality risk. In addition, compared with the absence of diabetes, the presence of diabetes and comorbid diseases synergistically increased mortality risk. Furthermore, the increased mortality risk was more prominent in the younger age groups than in the older age groups. Strict glycemic control and early intervention for comorbid cardiometabolic risk factors and diseases are warranted to attenuate the increased mortality risk in patients with diabetes.

Supplementary Materials: The supplementary materials are available online at http://www.mdpi.com/2077-0383/ 9/4/1042/s1.

Author Contributions: Each author has been involved in and contributed to this manuscript. Conceptualization, E.-J.R., Y.-G.P. and W.-Y.L.; Data curation, E.-J.R., K.-D.H. and Y.-G.P.; Formal analysis, K.-D.H. and Y.-G.P.; Writing-original draft, E.-J.R.; Writing-review \& editing, I.J., H.K., S.E.P., Y.-H.K. and W.-Y.L. All authors approved the final version of the manuscript to be published. 
Funding: This research received no external funding.

Acknowledgments: We would like to thank the Korean National Health Insurance Corporation and all the participants of the study and health check-up.

Conflicts of Interest: The authors declare no conflicts of interest.

\section{References}

1. Available online: https://diabetesatlas.org (accessed on 25 July 2019).

2. Kim, B.Y.; Won, J.C.; Lee, J.H.; Kim, H.S.; Park, J.H.; Ha, K.H.; Won, K.C.; Kim, D.J.; Park, K.S. Diabetes Fact Sheets in Korea, 2018: An Appraisal of Current Status. Diabetes. Metab. J. 2019, 43, 487-494. [CrossRef] [PubMed]

3. Rhee, E.J. Diabetes in Asians. Endocrinol. Metab. (Seoul) 2015, 30, 263-269. [CrossRef] [PubMed]

4. Zhang, Y.; Ning, G. Diabetes: Young-onset type 2 diabetes mellitus-A challenge for Asia. Nat. Rev. Endocrinol. 2014, 10, 703-704. [CrossRef] [PubMed]

5. Available online: https://www.who.int/news-room/fact-sheets/detail/the-top-10-causes-of-death (accessed on 25 July 2019).

6. American Diabetes Association. 2. Classification and Diagnosis of Diabetes: Standards of Medical Care in Diabetes-2019. Diabetes Care 2019, 42, S13-S28. [CrossRef]

7. Huang, Y.; Cai, X.; Mai, W.; Li, M.; Hu, Y. Association between prediabetes and risk of cardiovascular disease and all cause mortality: Systematic review and meta-analysis. BMJ 2016, 355, i5953. [CrossRef]

8. Ning, F.; Tuomilehto, J.; Pyörälä, K.; Onat, A.; Söderberg, S.; Qiao, Q.; DECODE Study Group. Cardiovascular disease mortality in Europeans in relation to fasting and $2 \mathrm{~h}$ plasma glucose levels within a normoglycemic range. Diabetes Care 2010, 33, 2211-2216. [CrossRef]

9. Markus, M.R.P.; Ittermann, T.; Baumeister, S.E.; Huth, C.; Thorand, B.; Herder, C.; Roden, M.; Siewert-Markus, U.; Rathmann, W.; Koenig, W.; et al. Prediabetes is associated with microalbuminuria, reduced kidney function and chronic kidney disease in the general population: The KORA (Cooperative Health Research in the Augsburg Region) F4-Study. Nutr. Metab. Cardiovasc. Dis. 2018, 28, $234-242$. [CrossRef]

10. Rhee, E.J.; Park, S.E.; Chang, Y.; Ryu, S.; Lee, W.Y. Baseline glycemic status and mortality in 241,499 Korean metropolitan subjects: A Kangbuk Samsung Health Study. Metabolism 2016, 65, 68-77. [CrossRef]

11. Diabetes Control and Complications Trial Research Group; Nathan, D.M.; Genuth, S.; Lachin, J.; Cleary, P.; Crofford, O.; Davis, M.; Rand, L.; Siebert, C. The effect of intensive treatment of diabetes on the development and progression of long-term complications in insulin-dependent diabetes mellitus. N. Engl. J. Med. 1993, 329, 977-986. [CrossRef]

12. UK Prospective Diabetes Study (UKPDS) Group. Effect of intensive blood-glucose control with metformin on complications in overweight patients with type 2 diabetes (UKPDS 34). Lancet 1998, 352, 854-865. [CrossRef]

13. Rawshani, A.; Rawshani, A.; Franzén, S.; Sattar, N.; Eliasson, B.; Svensson, A.M.; Zethelius, B.; Miftaraj, M.; McGuire, D.K.; Rosengren, A.; et al. Risk Factors, Mortality, and Cardiovascular Outcomes in Patients with Type 2 Diabetes. N. Engl. J. Med. 2018, 379, 633-644. [CrossRef] [PubMed]

14. Sattar, N.; Rawshani, A.; Franzén, S.; Rawshani, A.; Svensson, A.M.; Rosengren, A.; McGuire, D.K.; Eliasson, B.; Gudbjörnsdottir, S. Age at Diagnosis of Type 2 Diabetes Mellitus and Associations With Cardiovascular and Mortality Risks. Circulation 2019, 139, 2228-2237. [CrossRef] [PubMed]

15. Song, S.O.; Jung, C.H.; Song, Y.D.; Park, C.Y.; Kwon, H.S.; Cha, B.S.; Park, J.Y.; Lee, K.U.; KO, K.S.; Lee, B.W.; et al. Background and data configuration process of a nationwide population-based study using the korean national health insurance system. Diabetes. Metab. J. 2014, 38, 395-403. [CrossRef] [PubMed]

16. Levey, A.S.; Bosch, J.P.; Lewis, J.B.; Greene, T.; Rogers, N.; Roth, D. A more accurate method to estimate glomerular filtration rate from serum creatinine: A new prediction equation. Modification of Diet in Renal Disease Study Group. Ann. Intern. Med. 1999, 130, 461-470. [CrossRef] [PubMed]

17. Seo, M.H.; Lee, W.Y.; Kim, S.S.; Kang, J.H.; Kang, J.H.; Kim, K.K.; Kim, B.Y.; Kim, Y.H.; Kim, W.J.; Kim, E.M.; et al. 2018 Korean Society for the Study of Obesity Guideline for the Management of Obesity in Korea. J. Obes. Metab. Syndr. 2019, 28, 40-45. [CrossRef] [PubMed]

18. Available online: http://kosis.kr (accessed on 1 August 2019). 
19. Yang, J.J.; Yu, D.; Wen, W.; Saito, E.; Rahman, S.; Shu, X.O.; Chen, Y.; Gupta, P.C.; Gu, D.; Tsugane, S.; et al. Association of Diabetes with All-Cause and Cause-Specific Mortality in Asia: A Pooled Analysis of More Than 1 Million Participants. JAMA Netw. Open 2019, 2, e192696. [CrossRef] [PubMed]

20. Gaede, P.; Vedel, P.; Larsen, N.; Jensen, G.V.; Parving, H.H.; Pedersen, O. Multifactorial intervention and cardiovascular disease in patients with type 2 diabetes. N. Engl. J. Med. 2003, 348, 383-393. [CrossRef]

21. Emerging Risk Factors Collaboration; Di Angelantonio, E.; Kaptoge, S.; Wormser, D.; Willeit, P.; Butterworth, A.S.; Bansal, N.; O'Keeffe, L.M.; Gao, P.; Wood, A.M.; et al. Association of Cardiometabolic Multimorbidity With Mortality. JAMA 2015, 314, 52-60.

22. Home, P. Cardiovascular outcome trials of glucose-lowering medications: An update. Diabetologia 2019, 62, 357-369. [CrossRef]

23. Wright, A.K.; Kontopantelis, E.; Emsley, R.; Buchan, I.; Mamas, M.A.; Sattar, N.; Rutter, M.K.; Ashcroft, D.M. Life Expectancy and Cause-Specific Mortality in Type 2 Diabetes: A Population-Based Cohort Study Quantifying Relationships in Ethnic Subgroups. Diabetes Care 2017, 40, 338-435. [CrossRef]

24. Lin, C.C.; Li, C.I.; Liu, C.S.; Lin, W.Y.; Lin, C.H.; Chiang, J.I.; Yang, S.Y.; Li, T.C. Obesity paradox in associations between body mass index and diabetes-related hospitalization and mortality in patients with type 2 diabetes: Retrospective cohort studies. Diabetes Metab. 2019, 45, 564-572. [CrossRef] [PubMed]

25. Jung, H.H.; Park, J.I.; Jeong, J.S. Incidence of diabetes and its mortality according to body mass index in South Koreans aged 40-79 years. Clin. Epidemiol. 2017, 9, 667-678. [CrossRef] [PubMed]

26. Brown, J.C.; Harhay, M.O.; Harhay, M.N. Sarcopenia and mortality among a population-based sample of community-dwelling older adults. J Cachexia Sarcopenia Muscle 2016, 7, 290-298. [CrossRef] [PubMed]

27. Carnethon, M.R.; De Chavez, P.J.; Biggs, M.L.; Lewis, C.E.; Pankow, J.S.; Bertoni, A.G.; Golden, S.H.; Liu, K.; Mukamal, K.J.; Jenkins, B.C.; et al. Association of weight status with mortality in adults with incident diabetes. JAMA 2012, 308, 581-590. [CrossRef] [PubMed]

28. Perry, J.R.; Voight, B.F.; Yengo, L.; Amin, N.; Dupuis, J.; Ganser, M.; Grallert, H.; Navarro, P.; Li, M.; Qi, L.; et al. Stratifying type 2 diabetes cases by BMI identifies genetic risk variants in LAMA1 and enrichment for risk variants in lean compared to obese cases. PLoS Genet. 2012, 8, e1002741. [CrossRef] [PubMed]

29. Wing, R.R.; Lang, W.; Wadden, T.A.; Safford, M.; Knowler, W.C.; Bertoni, A.G.; Hill, J.O.; Brancati, F.L.; Peters, A.; Look AHEAD Research Group; et al. Benefits of modest weight loss in improving cardiovascular risk factors in overweight and obese individuals with type 2 diabetes. Diabetes Care 2011, 34, 1481-1486. [CrossRef]

(C) 2020 by the authors. Licensee MDPI, Basel, Switzerland. This article is an open access article distributed under the terms and conditions of the Creative Commons Attribution (CC BY) license (http://creativecommons.org/licenses/by/4.0/). 